\section{Cytokine loci co-localized and poised}

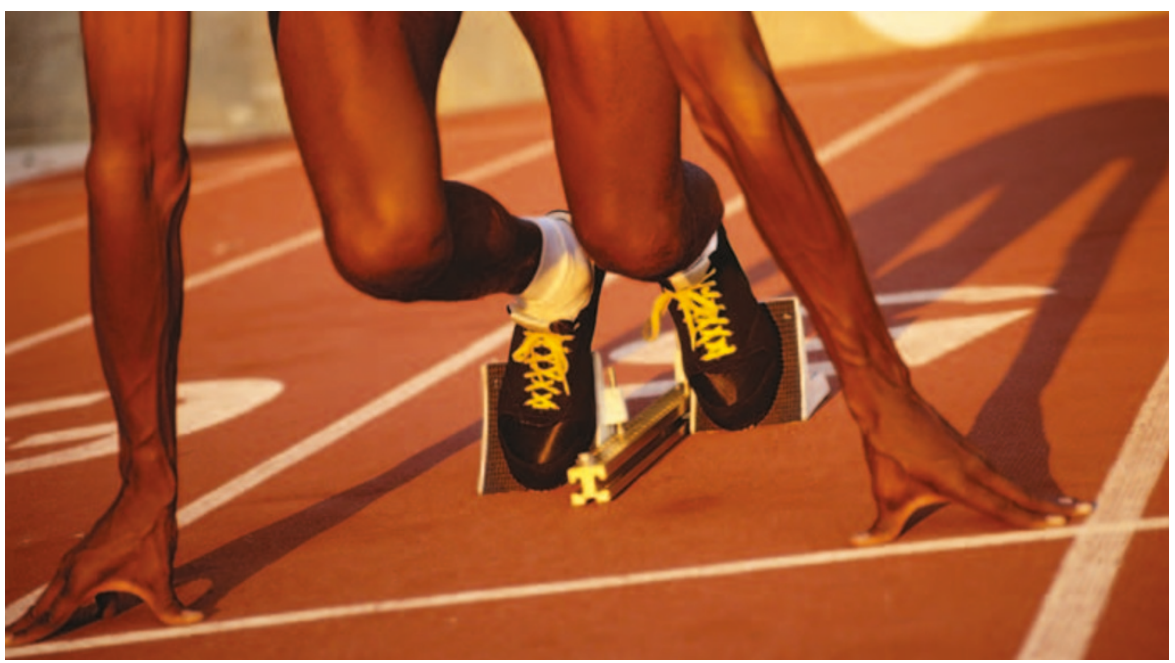

How are cytokine loci arranged in the nucleus to ensure coordinated and reciprocal expression of cytokine genes by $\mathrm{T}$ helper $1\left(\mathrm{~T}_{\mathrm{H}} 1\right)$ versus $\mathrm{T}_{\mathrm{H}} 2$ cells if they are present on different chromosomes? In a recent study in Nature, Richard Flavell and colleagues address this conundrum and provide the first direct evidence that cytokine loci on different chromosomes that are alternatively expressed by $\mathrm{T}_{\mathrm{H}}$-cell subsets are brought together in the nucleus, locating them in nuclear sites that are conducive to rapid expression in response to immune stimuli.
Expression of the $\mathrm{T}_{\mathrm{H}} 1$-cytokine gene interferon- $\gamma$ (Ifng) is regulated by elements near it on chromosome 10, whereas expression of the $\mathrm{T}_{\mathrm{H}}$ 2-cytokine genes interleukin-4 (Il-4), Il-5 and Il-13 on chromosome 11 are regulated by a locus control region (LCR) on the same chromosome, which controls expression of the entire $\mathrm{T}_{\mathrm{H}}$ 2-gene complex. But, using a recently developed chromosome-conformation capture technique, the authors could detect interchromosomal interactions between the promoter region of Ifng and the $\mathrm{T}_{\mathrm{H}} 2 \mathrm{LCR}$ in naive $\mathrm{T}_{\mathrm{H}}$ cells, which seems to add a further dimension to the regulation of these genes. After stimulation of naive cells to induce differentiation into $\mathrm{T}_{\mathrm{H}} 1$ or $\mathrm{T}_{\mathrm{H}} 2$ cells, the chromosomes seemed to move apart. Concomitant with loss of the interchromosomal interactions, in $\mathrm{T}_{\mathrm{H}} 1$ cells, intrachromosomal interactions between Ifng and its downstream regulatory element were favoured, indicating that the interactions are dynamic and cell-subset specific. These results were confirmed using fluorescence in situ hybridization (FISH).

The authors proposed that the purpose of such dynamic chromosome interactions was to position genetic loci in subnuclear compartments that poise and prepare these loci for rapid expression after stimulation. Consistent with this, deletion of an LCR regulatory element (RHS7), which disrupted interchromosomal associations, delayed the rapid induction of Ifng expression after stimulation under $\mathrm{T}_{\mathrm{H}} 1$-cell-promoting conditions. Similarly, under $\mathrm{T}_{\mathrm{H}}$ 2-cellpromoting conditions, cells that lacked RHS7 expressed less IL- 5 at early time points.

So, the $\mathrm{T}_{\mathrm{H}} 2 \mathrm{LCR}$ seems to regulate not only the transcription of the $\mathrm{T}_{\mathrm{H}} 2$-cytokine genes but also the expression of Ifng through mediating interchromosomal associations. Whether this phenomenon will be a common feature of other coordinately regulated genes awaits future research.

Lucy Bird

(2) References and links

ORIGINAL RESEARCH PAPER Spilianakis, C. G.,

Lalioti, M. D., Town, T., Lee, G. R. \& Flavell, R. A.

Interchromosomal associations between alternatively expressed loci. Nature 435, 637-645 (2005)

\title{
Driving diversity
}

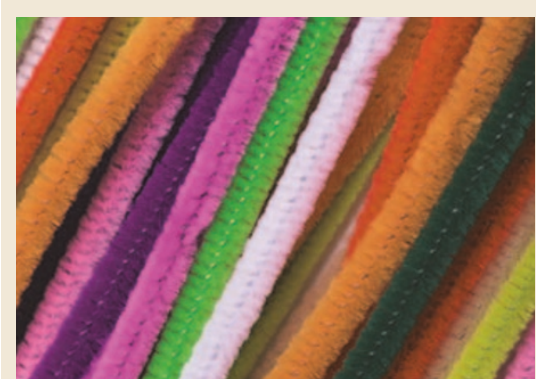

One explanation for the remarkable diversity of the MHC - which is the most polymorphic gene cluster in the human genome - is that genetic diversity is the result of pathogen-driven selection processes. Although there is some evidence indicating that this is the case, until now, there has been little direct evidence to support this idea.

If diversity is the result of pathogendriven selection, then one prediction would be that, in geographical regions of high pathogen diversity, humans should have increased diversity of HLA genes in relation to their average genomic diversity. To test this, the authors used 3 types of information: data on the genetic diversity of HLA class I molecules from 61 human populations; estimates of pathogen richness (that is, the total number of intracellular pathogens that are known to be present in each country); and the geographical distance of each population from East Africa (to control for the effect of past colonization history).
The authors found that human populations located further from East Africa had lower genetic variability and that human colonization history accounted for $17-39 \%$ of the diversity of HLA genes. The remaining diversity was significantly correlated with pathogen richness: that is, a population exposed to a more diverse range of pathogens has greater HLA diversity than one exposed to fewer pathogens. The results showed that pathogens (mainly viruses) exerted the greatest pressure on genes in the HLA-B locus; interestingly, it has recently been shown that HLA-B genes could have a larger role in containing viral infections than HLA-A genes.

These results show that human colonization history has been important in shaping the diversity of the HLA locus, but pathogen-driven selection has also had a role.

Elaine Bell

(2) References and links ORIGINAL RESEARCH PAPER Prugnolle, F. et al. Pathogen-driven selection and worldwide HLA class I diversity. Curr. Biol. 15, 1022-1027 (2005) 